\title{
Molecular Pathogenesis of Liver Steatosis Induced by Hepatitis C Virus
}

\author{
Jun Cheng, Min Li, Ping Gao, Jin-ling Dong and Qi Wang
}

Liver steatosis is a pathological hallmark in patients with chronic hepatitis C (CHC). Increased lipid uptake, decreased lipid secretion, increased lipid synthesis and decreased lipid degradation are all involved in pathogenesis of steatosis induced by hepatitic C virus (HCV) infection. Level of low density lipoprotein receptor (LDL-R) and activity of peroxisome proliferator-activated receptor (PPAR) $\alpha$ is related to liver uptake of lipid from circulation, and affected by HCV. Secretion via microsomal triglyceride transfer protein (MTTP), and formation of very low density lipoprotein (VLDL) have been hampered by HCV infection. Up-regulation of lipid synthesis related genes, such as sterol regulatory element-binding protein (SREBP)-1, SREBP-2, SREBP-1c, fatty acid synthase (FASN), HMG CoA reductase (HMGCR), liver X receptor (LXR), acetyl-CoA carboxylase 1 (ACC1), hepatic $\mathrm{CB}$ (1) receptors, retinoid $\mathrm{X}$ receptor (RXR) $\alpha$, were the main stay of liver steatosis pathogenesis. Degradation of lipid in liver is decreased in patients with CHC. There is strong evidence that heterogeneity of HCV core genes of different genotypes affect their effects of liver steatosis induction. A mechanism in which steatosis is involved in $\mathrm{HCV}$ life cycle is emerging.

Key words: Liver steatosis; Hepatitis C virus; Hepatitis; Lipid metabolism; Insulin resistance

$\mathrm{C}$ hronic hepatitis $\mathrm{C}(\mathrm{CHC})$ is characteristic for its liver steatosis. ${ }^{1}$ In order to replicate efficiently, structural and non-structural proteins of hepatitis $\mathrm{C}$ virus (HCV) bind with lipoproteins and apolipoproteins, and establish both macrovesicular and microvesicular fatty bubbles where HCV replication occurred. ${ }^{2}$ The pathogenesis of liver steatosis induced by $\mathrm{HCV}$ and its implications in the prevention and treatment of $\mathrm{CHC}$ remained largely unknown. ${ }^{3,4}$ Accumulated evidence indicated that the liver steatosis has complicated mechanism and important significance in the pathogenesis of $\mathrm{CHC}$ and $\mathrm{HCV}$ life cycle regulation. ${ }^{5}$

\section{PATHOGENESIS OF LIVER STEATOSIS INDUCED BY HCV INFECTION}

Accumulation of lipid in liver and formation of liver steatosis depended on the speed of lipid metabolism, such as uptake and secreation, synthesis and degradation, in the liver of patients with $\mathrm{CHC}^{6}$ After infection, structural and non-structural proteins of $\mathrm{HCV}$ affect these processes, and enhance the deposit of lipid in liver, and many transporters and key enzymes are involved in the pathogenesis of liver steatosis. ${ }^{7}$

\footnotetext{
Correspondence: Jun Cheng, Institute of Infectious Diseases, Beijing Ditan Hospital, Capital Medical University; 8 East Jingshun Street, Chaoyang District, Beijing 100015, China

Tel: +86-10-84322006; Fax: +86-10-84397196; E-mail: jun.cheng. ditan@gmail.com
}

\section{Increased uptake of lipid induced by $\mathrm{HCV}$}

Low density lipoprotein receptor (LDL-R) is the major receptor for lipid uptake from the circulation. HCV proteins upregulate the expression of LDL-R on the surface of hepatocyte to enhance the lipid uptake from blood circulation. On the other hand, peroxisome proliferator-activated receptor (PPAR) $\alpha$ is involved in the regulation of lipid intake in liver. Tanaka et al established PPAR $\alpha$-homozygous, -heterozygous, and -null mice with liver-specific transgenic expression of the HCV core protein gene Ppara $(+/+)$ : HCVcpTg, Ppara (+/-): HCVcpTg, and Ppara (-/-):HCVcpTg mice $^{8}$. Severe steatosis was unexpectedly observed only in Ppara $(+/+): H C V c p T g$ mice, which resulted from enhanced fatty acid uptake. These phenomena were found to be closely associated with sustained PPAR $\alpha$ activation. Long-term treatment of these mice with clofibrate, a PPAR activator, eventually induced hepatic steatosis. The results indicated that persistent activation of PPAR $\alpha$ is essential for the pathogenesis of hepatic steatosis induced by HCV infection. Single nucleotide polymorphism (SNP) of key regulator genes usually determine the effects in the pathogenesis of liver steatosis, such as SNP of interleukin-28B (IL28B) affects the formation of liver steatosis in patients with non-alcoholic fatty liver disease (NAFLD) and liver steatosis induced by HCV infection. But Verdi et al demonstrated that neither NASH nor genotype 1 HCV-related liver steatosis seemd to be associated with 
PPAR $\alpha$ L162V polymorphism. ${ }^{9}$

\section{Decreased secreation of lipid induced by $\mathrm{HCV}$}

HCV subverts host cholesterol metabolism for key processes in its life cycle, and this interference frequently results in genotype-dependent clinical sequelae of hypocholesterolemia. At the same time, hypocholesterolemia typically resolves after sustained viral response (SVR), implicating viral interference in host lipid secreation. Clark et al used a targeted cholesterol metabolic platform to evaluate paired $\mathrm{HCV}$ G2 and G3 patient sera for changes in in vivo HCV sterol pathway metabolites. ${ }^{10}$ In paired sera, baseline sterol levels were lower in G3 than G2 patients for distal metabolites such as 7-dehyrocholesterol (7DHC) and cholesterol, but not the proximal metabolite lanosterol. In HCV G3 patients, SVR resulted in increased levels of cholesterol, 7DHC. In contrast, lanosterol was unchanged after SVR. HCV G3, but not G2, selectively interferes with the late cholesterol synthesis pathway. Normal lanosterol levels provide a signal for the continued proteolysis of HMG CoA reductase, which may undermine other host responses to increase cholesterol synthesis. Yamaguchi et al found hepatic TG content and hepatic thiobarbituric acid-reactive substances increased. PPAR $\alpha$, MDR2, acyl-CoA oxidase (AOX) and carnitine palmitoyl transferase-1 (CPT-1) were down-regulated in HCV core protein-expressing mice. HCV core protein downregulated lipid metabolism-associated gene expression, MDR2, CPT and AOX, accompanied by downregulation of PPAR $\alpha$. These findings may contribute to understanding the decreased secreation of lipid from the liver.

Among the structural proteins of HCV, Perlemuter et al demonstrated core protein inhibits MTTP activity and very low density lipoprotein (VLDL) secretion. ${ }^{11}$ In the transgenic murine model expressing core protein of $\mathrm{HCV}$, overexpression of $\mathrm{HCV}$ core protein interferes with the hepatic assembly and secretion of triglyceride-rich VLDL. Core protein expression led to reduction in MTTP activity and in the particle size of nascent hepatic VLDL without affecting accumulation of MTTP and protein disulfide isomerase. Hepatic human apolipoprotein A II (apo A II) expression in double-core/apo A II transgenic mice diminished intrahepatic core protein accumulation and abrogated its effects on VLDL production. Apo A II and HCV core colocalized in $\mathrm{HCV}$-infected liver biopsies, thus testifying to the relevance of this interaction in productive HCV infection. The results lead to propose a new pathophysiological animal model for induction of viral-related steatosis whereby the core protein of $\mathrm{HCV}$ targets microsomal triglyceride transfer protein (MTTP) activity and modifies hepatic VLDL assembly and secretion.

MTTP is required for assembly and secretion of Apo $\mathrm{B}$ lipoprotein, and its activity determines the efficiency of secreation of lipid from liver. HCV impaired MTTP activity resulting in significant reduction of transfer of lipid from liver. So liver steatosis caused by HCV infection is also called a storage liver disease. ${ }^{12}$ Higher degrees of hepatic steatosis were found in chronic hepatitis $\mathrm{C}$ patients carrying the $\mathrm{T}$ allele of MTTP $-493 \mathrm{G} / \mathrm{T}$ polymorphism that seems to be associated with increased MTTP transcription.

In a transgenic mouse model, $\mathrm{HCV}$ was shown to inhibit MTTP activity, which is essential for hepatic lipoprotein assembly and secretion. Mirandola et al investigated liver MTTP gene expression and its lipid transfer activity in untreated cases infected with the major HCV genotypes. ${ }^{12}$ They found that MTTP mRNA levels showed a statistically significant inverse correlation with the degree of steatosis, independently of the HCV genotype. MTTP mRNA levels also had an inverse correlation with serum insulin, homeostasis model assessment-insulin resistance (HOMA-IR) in patients with HCV G1 and HCV G2 and with serum HCV-RNA in HCV G3 infected patients. Liver MTTPspecific activity was significantly reduced in HCV G3 infected patients compared with other HCV genotypes infected patients and correlated with reduced serum cholesterol, apo B and low-density lipoproteins.

Ryan et al measured the expression of genes involved in lipid metabolism including MTTP in liver biopsies from patients infected with HCV genotype- 1 and $-3 .{ }^{13} \mathrm{~A}$ significant genotype-independent correlation was found among the expression of Apo B, MTTP, PRKAA1 and PPAR $\alpha$, indicating that these networks are functional in $\mathrm{HCV}$-infected liver.

Mirandola et al genotyped for MTTP -493G/T variants in two hundred and ninety-eight $\mathrm{CHC}$ patients and found grades $2 / 3$ steatosis patients had lower MTTP mRNA expression. ${ }^{14}$ MTTP T allele was an independent risk factors for steatosis grades $2 / 3$, and in HCV genotype non-3 patients, the MTTP T allele was the strongest predictor for steatosis grade $2 / 3$. Zampino et al also found that MTTP $-493 \mathrm{G} / \mathrm{T}$ gene polymorphism contributed to fat liver accumulation in HCV genotype-3 infected patients. ${ }^{15}$ But Petit et al found lack of association between MTTP gene polymorphism and liver steatosis in $\mathrm{HCV}$-infected patients. ${ }^{16}$

\section{Increased synthesis of lipid induced by HCV SREBP family members in steatosis}

Sterol regulatory element binding protein (SREBP) family consists of multiple members including SREBP-1c, SREBP-1 and SREBP-2, which are major regulators of lipid metabolism. Kim et al determined 
whether HCV core protein may impair the expression and activity of SREBP. ${ }^{17}$ It was demonstrated that $\mathrm{HCV}$ core protein increases the gene expression of SREBP-1. Furthermore, HCV core protein enhanced the transcriptional activity of SREBP-1. Jackel-Cram et al showed that HCV-3a core protein upregulated the promoter activity of FASN through SREBP-1. ${ }^{18}$ Their results also showed that the level of mature SREBP-1 was enhanced by HCV-3a core protein. By using the chemical inhibitor LY294002, a dominantnegative Akt-2 plasmid, or knock-down of Akt-2 by small hairpin RNA, the inhibition of PI3K and Akt-2 was associated with reduced SREBP-1 activation by HCV-3a core protein. Park et al demonstrated that HCV NS4B protein increased the transcriptional activities of both SREBP and fatty acid synthetase (FASN) requiring phosphatidylinositol 3-kinase (PI3K) activity. ${ }^{19}$ In addition, NS4B protein synergistically elevated the transcriptional activity of HCV coremediated SREBP-1. Xiang et al showed that the nuclear, mature SREBP-1c level increased in the nucleus of replicon cells expressing HCV-3a NS5A. ${ }^{20}$ Additional analysis showed that transcriptional factor $\mathrm{Sp} 1$ is involved in SREBP-1c activation by HCV-3a NS5A. McPherson et al found no significant difference in the hepatic expression of SREBP-1c mRNA between subjects with HCV infection and normal liver. ${ }^{21}$ In patients with $\mathrm{HCV}$ infection, a significantly negative relationship was found between hepatic SREBP$1 \mathrm{c}$ mRNA expression and grades of steatosis, stages of fibrosis and severity of inflammation. Waris et al determined whether genotype 2a-based HCV infection induces the expression and posttranslational activation of SREBPs. ${ }^{22}$ The studies showed that HCV-induced oxidative stress and subsequent activation of PI3KAkt pathway and inactivation (phosphorylation) of PTEN (phosphatase and tensin homologue) mediated the transactivation of SREBPs. HCV-induced SREBP-1 and -2 activities were sensitive to antioxidant.

\section{FASN in steatosis}

Fatty acid synthetase is a key enzyme in fatty acid synthesis. In the liver of patients with CHC, FASN activity was up-regulated to enhance the synthesis of fatty acid. Jackel-Cram et al demonstrated that both HCV-3a and $-1 b$ core proteins up-regulated the FASN promoter, and found that FASN up-regulation by HCV core was dependent on SREBP- $1 .{ }^{23}$ Mutational analysis showed that processing of $\mathrm{HCV}$ core protein of different genotypes was differentially involved in FASN promoter up-regulation. Although lipid droplet localization of HCV core protein was not important for FASN up-regulation, a specific amino acid residue [Phe(164)] within the FATG lipid droplet localization sequence of HCV-3a core protein played a major role in the stronger FASN activation by HCV-3a core. Oem et al showed that HCV NS2 protein activated SREBP1c transcription, and sterol regulatory element (SRE) and liver X receptor element (LXRE) in the SREBP1c promoter were involved in SREBP-1c activation by HCV NS2. ${ }^{24}$ Furthermore, expression of HCV NS2 resulted in the upregulation of FASN transcription. FASN upregulation by HCV NS2 was SREBP-1dependent. The results suggest that HCV NS2 can upregulate the transcription of SREBP-1c and FASN, and thus is probably a contributing factor for HCVassociated steatosis.

\section{HMG CoA in steatosis}

HMG-CoA reductase (HMGCR) is the rate-limiting enzyme in the cholesterol biosynthetic pathway. In clinical practice, HMGCR is the target for statins. Honda et al reviewed the roles of cholesterol in $\mathrm{HCV}$ infection and introduces $\mathrm{HCV}$ events related to cholesterol metabolism and applications for cholesterol metabolism as a therapeutic target. ${ }^{25} \mathrm{HCV}$ appears to alter host lipid metabolism into its preferable state, which is clinically recognized as steatosis and hypocholesterolemia. While hepatic fatty acid and triglyceride syntheses are upregulated in chronic hepatitis $\mathrm{C}$ patients, no direct evidence of increased hepatic de novo cholesterol biosynthesis has been obtained. Impaired VLDL secretion from hepatocytes is suggested to increase intracellular cholesterol concentrations, which may lead to hypocholesterolemia. Clinically, lower serum cholesterol levels are associated with lower rates of sustained virological responses (SVR) to pegylated-interferon plus ribavirin therapy, but the reason remains unclear. Anti-HCV actions by statins appear to be caused by the inhibition of geranylgeranyl pyrophosphate synthesis rather than their cholesterol lowering effects. Other compounds that block various steps of cholesterol metabolic pathways also have been studied to develop new strategies for the complete eradication of this virus.

\section{$P P A R$ in liver steatosis}

Kim et al found that NS5A protein increases hepatic lipid accumulation via induction of activation and expression of PPAR $\gamma$. The NS5A augmented the transcriptional activity and gene expression of PPAR $\gamma$. Furthermore, NS5A increased the ability to recruit the transcriptional coactivator PGC-1s to the PPRE with $\operatorname{PPAR} \gamma$, as well as the interaction with PPAR $\gamma 2$ and PGC-1 $\alpha$. The results indicated that NS5A may exploit multiple strategies that enhance PPAR $\gamma$-induced lipid accumulation. ${ }^{26}$

\section{LXR in steatosis}

Lima-Cabello et al reported that, besides inducing hepatic fatty acid biosynthesis, liver X receptor (LXR) regulated a set of inflammatory genes. ${ }^{27}$ They evaluated the hepatic expression of LXR $\alpha$ and its lipogenic and inflammatory targets in $44 \mathrm{CHC}$ patients, and found 
LXR $\alpha$ gene and its lipogenic targets PPAR $\gamma$, SREBP $-1 \mathrm{c}$, SREBP-2 and FASN were overexpressed in the liver of HCV infected patients who had steatosis. Hepatic expression of LXR $\alpha$ and its related lipogenic and inflammatory genes is abnormally increased in $\mathrm{HCV}$ infected patients with steatosis, suggesting a potential role of LXR $\alpha$ in the pathogenesis of hepatic steatosis.

Moriishi et al showed that a knockout of PA28 $\gamma$ gene induced the accumulation of $\mathrm{HCV}$ core protein in the nucleus of hepatocytes of CoreTg mice and disrupted the development of both hepatic steatosis and $\mathrm{HCC} \cdot{ }^{28}$ Furthermore, the genes related to fatty acid biosynthesis and srebp-1c promoter activity were upregulated by $\mathrm{HCV}$ core protein in cell line and mouse liver in a PA28 $\gamma$-dependent manner. Heterodimer composed of LXR $\alpha$ and retinoid X receptor (RXR) $\alpha$ is known to up-regulate srebp-1c promoter activity. The data also showed that HCV core protein enhanced the binding of LXR $\alpha /$ RXR $\alpha$ to LXR-response element in the presence but not the absence of PA2 8 . These findings suggested that PA2 $\gamma \gamma$ played a crucial role in the development of liver steatosis induced by $\mathrm{HCV}$ infection.

\section{$R X R \alpha$ in steatosis}

Retinoid X receptor $\alpha$, a transcriptional regulator that controls many aspects of cell proliferation, differentiation and lipid metabolism. Tsutsumi et al reported the direct interaction of $\mathrm{HCV}$ core protein with RXR $\alpha .{ }^{29}$ The core protein binds to the DNA-binding domain of RXR $\alpha$, leading to increase of DNA binding of RXR $\alpha$ to its responsive element. In addition, RXR $\alpha$ is activated in cells expressing core protein as well as in the livers of the core-transgenic mice that would develop hepatic steatosis and HCC later in their livers. Using promoter genes of cellular retinol binding protein II (CRBP II ) and acyl-CoA oxidase as reporters, they also showed that the expression of core protein enhanced the transcriptional activity regulated by the RXR $\alpha$ homodimer as well as by the heterodimer with peroxisome PPAR $\alpha$. Furthermore, expression of the CRBP II gene is also up-regulated in livers of $\mathrm{HCV}$ core-transgenic mice.

\section{PA28 $\gamma$ in steatosis}

The nuclear proteasome activator PA28 $\gamma /$ REG $\gamma$, which specifically binds to the core protein, is required for the virulence of the core protein. Elucidation of the mechanisms by which $\mathrm{HCV}$ core protein participates in the above conditions may provide clues toward the development of novel therapeutic measures for chronic hepatitis $\mathrm{C}^{30}$

\section{ACC1 in steatosis}

Fukasawa et al found that enhancement of fatty acid biosynthesis in core-expressing cells was caused by increased expression of fatty acid biosynthetic enzymes, especially ACC1. Up-regulation of de novo fatty acid biosynthesis by HCV core protein may affect cellular lipid metabolism, resulting in neutral lipid accumulation in $\mathrm{HCV}$-infected cells. ${ }^{31}$

\section{$C B$ (1) in steatosis}

Previous study results indicated that activation of hepatic CB (1) receptors is associated with steatosis and fibrosis in experimental forms of liver disease. van der Poorten et al measured CB (1) receptor mRNA on extracted liver tissue from patients with $\mathrm{CHC}$, and Huh7/JFH1 hepatitis C virus (HCV) cell culture model. They found that $\mathrm{CB}$ (1) was expressed in all patients with $\mathrm{CHC}$, and $\mathrm{CB}$ (1) expression increased with fibrosis stage increasing. Even in mild $\mathrm{CHC}$ with no steatosis, CB (1) levels remained substantially. Huh7 cells infected with JFH-1 HCV showed significant upregulation of $\mathrm{CB}$ (1), suggesting that $\mathrm{CB}$ (1) was an HCV-inducible gene. Although HCV structural proteins appear essential for CB (1) induction, there was no core genotype specific difference in CB (1) expression. CB (1) increased significantly with steatosis grade increasing, primarily driven by patients with genotype 3 CHC. In genotype 3 patients, CB (1) correlated with SREBP-1c and its downstream target FASN. So it is belived that $\mathrm{CB}$ (1) is up-regulated in $\mathrm{CHC}$ and is associated with increased steatosis in $\mathrm{HCV}$ genotype 3. ${ }^{32}$

\section{$\delta$-9 desaturase in steatosis}

Disturbance in lipid metabolism is one of the features of chronic hepatitis $\mathrm{C}$, being a crucial determinant of the progression of liver fibrosis. Miyoshi et al determined activities of fatty acid metabolizing enzymes by analyzing the fatty acid compositions in HepG2 cells with or without core protein expression. ${ }^{33}$ They found a marked accumulation of triglycerides in core-expressing HepG2 cells. While the oleic/stearic acid (18:1/18:0) and palmitoleic/palmitic acid ratio (16:1/16:0) were comparable in both core-expressing and control cells, and there was a marked accumulation of downstream product, 5,8,11-eicosatrienoic acid [20:3 (n-9)] in the core-expressing HepG2 cells. The addition of eicosatetraynoic acid, which inhibits delta- 6 desaturase activity that is inherently high in HepG2 cells, led to a marked accumulation of oleic and palmitoleic acids in the core-expressing cells, showing that $\delta-9$ desaturase was activated by the core protein. Eicosapentaenoic acid [20:5 (n-3)] or arachidonic acid [20:4 (n-6)] administration decreased $\delta-9$ desaturase activity significantly, with the concentration of 20:3 (n-9) and triglyceride accumulation. This lipid metabolism disorder was associated with NADH accumulation due to mitochondrial dysfunction, and was reversed by the addition of pyruvate through 
NADH utilization. The fatty acid enzyme $(\delta-9$ desaturase) was activated by HCV core protein and polyunsaturated fatty acids counteracted this impact of the core protein on lipid metabolism. These results may open up new insights into the mechanism of lipid metabolism disorder associated with $\mathrm{HCV}$ infection and provide clues for the development of new therapeutic devices.

\section{Decreased degradation of lipid induced by $\mathrm{HCV}$}

Hepatic fat export occurs through apolipoprotein B-100-containing lipoprotein production, whereas impaired production leads to liver steatosis. HCV infection is associated with dysregulation of apo B-100 secretion and steatosis. Mancone et al combinesd quantitative proteomics and computational biology, to propose ferritin heavy chain (Fth) as the cellular determinant of apo B-100 production inhibition..$^{34}$ They found that HCV non-structural proteins and NS5A appeared to be sufficient for inducing Fth up-regulation. Fth in turn was found to inhibit apo B-100 secretion which led to increased intracellular degradation via proteasome. Notably, intracellular Fth down-regulation by siRNA restores apo B-100 secretion. The inverse correlation between ferritin and plasma apo B-100 concentrations was also found in JFH-1 HCV cell culture systems (HCVcc) and $\mathrm{HCV}$-infected patients. Finally, Fth expression was found to be required for robust $\mathrm{HCV}$ infection. These observations provide a further molecular explanation for the onset of liver steatosis and allow for hypothesizing on new therapeutic and antiviral strategies.

\section{SEQUENCE HETEROGENEITY OF HCV AND LIVER STEATOSIS}

Polymorphism of HCV core gene in different genotypes is important for its ability to induce liver steatosis. In genotype $1 \mathrm{~b} \mathrm{HCV}$ infected patients, amino acid substitutions in core region impact on liver steatosis. Tachi et al analyzed HCV genotype $1 \mathrm{~b}$ core region sequences from 67 patients with $\mathrm{CHC}^{35}$ Substitutions of amino acid 70/Q (glutamine) were significantly associated with the presence of steatosis on univariate analysis. Multivariate analysis showed that substitution of amino acid 70 of glutamine and triglyceride levels were the independent factors related to liver steatosis. Hepatic and urinary $8-\mathrm{OHdG}$ levels were significantly higher in patients with methionine at amino acid 91 of the HCV core region than in those with leucine. Akuta et al evaluated the impact of amino acid (aa) substitutions of HCV-1b core region on IR in 123 Japanese patients infected with HCV-1b without cirrhosis and diabetes mellitus, and investigated the treatment efficacy of 48-week pegylated interferon (PEG-IFN) plus ribavirin (RBV) according to HOMAIR values. ${ }^{36}$ The study indicated that substitutions of HCV-1b core region were the important predictor of severe IR in patients without cirrhosis and diabetes mellitus, but HOMA-IR values might be not useful as predictors of 48-week PEG-IFN plus RBV therapy. Sumida et al found substitutions of amino acid 70 were significantly associated with the presence of steatosis, upon univariate analysis in patients with HCV genotype $1 \mathrm{~b}$ infection. ${ }^{37}$

In HCV genotype-3 infected patients, the etiology of steatosis appears to be closely correlated with unknown viral factors that increase intracellular lipid levels. Depla et al analyzed the possible association of particular residues of the core protein of genotype-3 $\mathrm{HCV}$ and dysregulate lipid metabolism with steatosis severity. ${ }^{38}$ Transmission electron microscopy found the core protein sequences of the viral variants circulating in patients with mild or severe steatosis were evenly distributed throughout the phylogenic trees indicating the host factors occupy more important role in the pathogenesis of liver steatosis. Jhaveri et al found two polymorphisms at positions 182 and 186 of the core protein correlated with the presence and absence of intrahepatic steatosis. ${ }^{39}$ Hourioux et al demonstrated that lipid droplet area was significantly greater in sections of cells producing the Y164F mutant HCV core protein than in cells producing the wild-type protein. ${ }^{40}$ The frequency of cell sections containing lipid droplets, in particular, was higher for the mutant than for the wild-type protein.

\section{POLYMORPHISM OF IL-28B GENE AND LIVER STEATOSIS}

From genome wide association study (GWAS) of patients with $\mathrm{CHC}$ treated with pegylated interferon plus ribavirin, it is found that the polymorphism of IL-28B gene of the host is a determining parameter at baseline for SVR. Presently IL-28B polymorphisms have been associated with both treatment induced and spontaneous clearance of HCV. The biological implications of polymorphism in IL-28B gene and the relevance to the SVR of CHC patients to SOC remain unclear. Recently, an association between $\gamma$-GTP level and IL-28B genotype was identified. Cai et al evaluated the important single nucleotide polymorphisms (SNPs) of important molecular in the pathogenesis of liver steatosis of CHC patients, such as those in the patatin-like phospholipase family 3 protein (PNPLA3), IL-28B, etc, was never evaluated. ${ }^{41}$ After analysis of 626 Caucasian HCV infected patients, SNPs were extracted from a genome-wide association- 
generated dataset. Associations of alleles with the presence and/or different severity of steatosis were evaluated by univariate and multivariate logistic regression, accounting for all relevant covariates. The risk of steatosis was increased by carriage of $1148 \mathrm{M}$ in PNPLA3, but only in patients with HCV genotypes non-3 and similar, albeit weaker associations were found for SNPs in PPAR $\gamma$ and IL-28B. Carriage of a SNP in the MTTP increased the risk of steatosis, but only in patients with HCV genotype 3. So they thought that the rs738409 SNP in PNPLA3 was associated with an increased risk of steatosis in patients infected with HCV genotypes non-3. Host genes including PNPLA3, PPAR $\gamma$ and IL-28B, affect steatosis depending on the infecting $\mathrm{HCV}$ genotype, suggesting their interaction with viral factors.

Ohnishi et al also found IL-28B polymorphism was associated with fatty change in the liver of chronic hepatitis $\mathrm{C}$ patients. ${ }^{42}$ Vesicular change in the liver tissue was significantly associated with body mass index (BMI), HCV RNA titer, serum aspartate aminotransferase, $\gamma$-GTP, IL-28B genotype and liver fibrosis level. Clear cell change was significantly associated with serum aspartate aminotransferase, $\gamma$-GTP and IL-28B genotype. IL-28B genotype, liver fibrosis and BMI were significantly independent factors for vesicular change and IL-28B genotype for clear cell change.

Tillmann et al firstly found that LDL cholesterol levels were higher in $\mathrm{CHC}$ patients with the $\mathrm{CC}$ genotype at the rs 12979860 polymorphism, located proximal to the IL-28 gene, and then analyzed the association of steatosis with IL-28B genotype in treatment of naïve patients with $\mathrm{CHC}{ }^{43}$ Total of 325 CHC patients in two cohorts were analyzed for the presence and severity of steatosis in relation to the rs12979860 polymorphism at the IL-28B locus. CC genotype was associated with a lower prevalence of steatosis. Steatosis was found in $47.6 \%$ IL-28B nonCC vs. $22.5 \%$ in CC patients in the first cohort of patients. Similarly, steatosis was found in $67.4 \%$ nonCC patients compared with only $39.6 \%$ of CC patients in the second cohort. These results indicated that IL28B CC genotype was associated with less pronounced disturbances of lipid metabolism, as reflected both in serum lipoprotein levels and hepatic steatosis, in HCV infection.

\section{LIVER STEATOSIS AND HCV LIFE CYCLE REGULATION}

$\mathrm{HCV}$ infection is associated with dysregulation of both lipid and glucose metabolism. As well as contributing to the pathogenesis of steatosis, insulin resistance and type 2 diabetes, these perturbations influence the viral replication. In another word, HCV induces liver steatosis to enhance its replication. ${ }^{44-48}$

AMP-activated protein kinase (AMPK) plays a key role in regulation of both lipid and glucose metabolism. Mankouri et al showed that, in cells either infected with HCV or harboring an HCV subgenomic replicon, phosphorylation of AMPK at threonine 172 and concomitant AMPK activity were dramatically reduced. ${ }^{49}$ They demonstrated that this effect was mediated by activation of the serine/threonine kinase, protein kinase B, which inhibits AMPK by phosphorylating serine 485 . The physiological significance of this inhibition is demonstrated by the observation that pharmacological restoration of AMPK activity not only abrogates the lipid accumulation observed in virus-infected and subgenomic repliconharboring cells, but also efficiently inhibits viral replication. These data demonstrate that inhibition of AMPK is required for HCV replication.

Yang et al identified 175 proteins from a cell culture supernatant fraction containing the HCV genotype 2a (JFH-1) virus, among which FASN was highly enriched. ${ }^{50}$ Subsequent studies showed that FASN expression increased in Huh7 cell line. Blocking FASN activity by $\mathrm{C} 75$, a pharmacological FASN inhibitor, led to decreased HCV production. Reduction of FASN by RNA interference suppressed viral replication in both replicon and infection systems. Remarkably, FASN appeared to be selectively required for the expression of claudin-1, a tight junction protein that was recently identified as an entry coreceptor for $\mathrm{HCV}$, but not for the expression of another HCV coreceptor, CD81. The decrease in Claudin-1 expression resulting from FASN inhibition was accompanied by a decrease in transepithelial electric resistance of Huh7 cells, implying a reduction in the relative tightness of the cell monolayer. Consequently, the entry of human immunodeficiency virus-HCV pseudotypes was significantly inhibited in C75-treated Huh7 cells. As far as we know, this is the first line of evidence which demonstrates that $\mathrm{HCV}$ infection directly induces FASN expression, and thus suggests a possible mechanism by which HCV infection alters the cellular lipid profile and causes diseases such as steatosis. ${ }^{51,52}$

\section{REFERENCES}

1. Chang ML, Yeh HC, Tsou YK, Wang CJ, Cheng HY, Sung CM, et al. HCV core-induced nonobese hepatic steatosis is associated with hypoadiponectinemia and is ameliorated by adiponectin administration. Obesity (Silver Spring) 2012; doi: 10.1038/ oby.2012.45.

2. Sato S, Fukasawa M, Yamakawa Y, Natsume T, Suzuki T, Shoji $\mathrm{I}$, et al. Proteomic profiling of lipid droplet proteins in hepatoma 
cell lines expressing hepatitis $\mathrm{C}$ virus core protein. J Biochem 2006;139:921-930.

3. Congiu M, Ryan MC, Desmond PV. No increase in the expression of key unfolded protein response genes in HCV genotype 3 patients with severe steatosis. Virus Res 2011;160:420-423.

4. Congiu M, Slavin JL, Desmond PV. Expression of common housekeeping genes is affected by disease in human hepatitis $\mathrm{C}$ virus-infected liver. Liver Int 2011;31:386-390.

5. Khan M, Jahan S, Khaliq S, Ijaz B, Ahmad W, Samreen B, et al. Interaction of the hepatitis $\mathrm{C}$ virus $(\mathrm{HCV})$ core with cellular genes in the development of HCV-induced steatosis. Arch Virol 2010;155:1735-1753.

6. Depla M, d'Alteroche L, Le Gouge A, Moreau A, Hourioux C, Meunier JC, et al. Viral sequence variation in chronic carriers of hepatitis $\mathrm{C}$ virus has a low impact on liver steatosis. PLoS One 2012;7(3):e33749.

7. Ivanov AV, Smirnova OA, Ivanova ON, Masalova OV, Kochetkov $\mathrm{SN}$, Isaguliants MG. Hepatitis C virus proteins activate NRF2/ ARE pathway by distinct ROS-dependent and independent mechanisms in HUH7 cells. PLoS One 2011;6:e24957.

8. Tanaka N, Moriya K, Kiyosawa K, Koike K, Gonzalez FJ, Aoyama T. PPARalpha activation is essential for HCV core protein-induced hepatic steatosis and hepatocellular carcinoma in mice. J Clin Invest 2008;118:683-694.

9. Verdi H, Koytak ES, Onder O, Ergül AA, Cinar K, Idilman R, et al. Peroxisome proliferator-activated receptor alpha L162V polymorphism in nonalcoholic steatohepatitis and genotype 1 hepatitis C virus-related liver steatosis. J Investig Med 2005;53:353-359.

10. Clark P, Thompson A, Vock D, Kratz L, Tolun A, Muir A, et al. Hepatitis $\mathrm{C}$ virus selectively perturbs the distal cholesterol synthesis pathway in a genotype specific manner. Hepatology 2012; doi: 10.1002/hep.25631.

11. Perlemuter G, Sabile A, Letteron P, Vona G, Topilco A, Chrétien $\mathrm{Y}$, et al. Hepatitis $\mathrm{C}$ virus core protein inhibits microsomal triglyceride transfer protein activity and very low density lipoprotein secretion: a model of viral-related steatosis. FASEB J 2002;16:185-194.

12. Mirandola S, Osterreicher CH, Marcolongo M, Datz C, Aigner E, Schlabrakowski A, et al. Microsomal triglyceride transfer protein polymorphism $(-493 \mathrm{G} / \mathrm{T})$ is associated with hepatic steatosis in patients with chronic hepatitis C. Liver Int 2009;29:557-565.

13. Ryan MC, Desmond PV, Slavin JL, Congiu M. Expression of genes involved in lipogenesis is not increased in patients with HCV genotype 3 in human liver. J Viral Hepat 2011;18:53-60.

14. Mirandola S, Realdon S, Iqbal J, Gerotto M, Dal Pero F, Bortoletto $\mathrm{G}$, et al. Liver microsomal triglyceride transfer protein is involved in hepatitis C liver steatosis. Gastroenterology 2006;130:1661-1669.

15. Zampino R, Ingrosso D, Durante-Mangoni E, Capasso R, Tripodi MF, Restivo L, et al. Microsomal triglyceride transfer protein (MTP) $-493 \mathrm{G} / \mathrm{T}$ gene polymorphism contributes to fat liver accumulation in HCV genotype 3 infected patients. J Viral Hepat 2008;15:740-746.

16. Petit JM, Masson D, Minello A, Duvillard L, Galland F, Verges $\mathrm{B}$, et al. Lack of association between microsomal triglyceride transfer protein gene polymorphism and liver steatosis in HCVinfected patients. Mol Genet Metab 2006;88:196-198.

17. Kim K, Kim KH, Kim HY, Cho HK, Sakamoto N, Cheong J. Curcumin inhibits hepatitis $C$ virus replication via suppressing the Akt-SREBP-1 pathway. FEBS Lett 2010;584:707-712.

18. Jackel-Cram C, Qiao L, Xiang Z, Brownlie R, Zhou Y, Babiuk $\mathrm{L}$, et al. Hepatitis $\mathrm{C}$ virus genotype-3a core protein enhances sterol regulatory element-binding protein-1 activity through the phosphoinositide 3-kinase-Akt-2 pathway. J Gen Virol 2010;91:1388-1395.

19. Park CY, Jun HJ, Wakita T, Cheong JH, Hwang SB. Hepatitis $\mathrm{C}$ virus nonstructural $4 \mathrm{~B}$ protein modulates sterol regulatory element-binding protein signaling via the AKT pathway. J Biol Chem 2009;284:9237-9246.

20. Xiang Z, Qiao L, Zhou Y, Babiuk LA, Liu Q. Hepatitis C virus nonstructural protein-5A activates sterol regulatory element- binding protein-1c through transcription factor Sp1. Biochem Biophys Res Commun 2010;402:549-553.

21. McPherson S, Jonsson JR, Barrie HD, O'Rourke P, Clouston $\mathrm{AD}$, Powell EE. Investigation of the role of SREBP-1c in the pathogenesis of HCV-related steatosis. J Hepatol 2008;49:1046-1054.

22. Waris G, Felmlee DJ, Negro F, Siddiqui A. Hepatitis C virus induces proteolytic cleavage of sterol regulatory element binding proteins and stimulates their phosphorylation via oxidative stress. J Virol 2007;81:8122-8130.

23. Jackel-Cram C, Babiuk LA, Liu Q. Up-regulation of fatty acid synthase promoter by hepatitis $\mathrm{C}$ virus core protein: genotype3 a core has a stronger effect than genotype-1b core. J Hepatol 2007;46:999-1008.

24. Oem JK, Jackel-Cram C, Li YP, Zhou Y, Zhong J, Shimano H, et al. Activation of sterol regulatory element-binding protein $1 \mathrm{c}$ and fatty acid synthase transcription by hepatitis $\mathrm{C}$ virus non-structural protein 2. J Gen Virol 2008;89:1225-1230.

25. Honda A, Matsuzaki Y. Cholesterol and chronic hepatitis $\mathrm{C}$ virus infection. Hepatol Res 2011;41:697-710.

26. Kim K, Kim KH, Ha E, Park JY, Sakamoto N, Cheong J. Hepatitis $\mathrm{C}$ virus NS5A protein increases hepatic lipid accumulation via induction of activation and expression of PPARgamma. FEBS Lett 2009;583:2720-2726.

27. Lima-Cabello E, García-Mediavilla MV, Miquilena-Colina ME, Vargas-Castrillón J, Lozano-Rodríguez T, Fernández-Bermejo M, et al. Enhanced expression of pro-inflammatory mediators and liver X-receptor-regulated lipogenic genes in non-alcoholic fatty liver disease and hepatitis C. Clin Sci (Lond) 2011;120:239-250.

28. Moriishi K, Mochizuki R, Moriya K, Miyamoto H, Mori Y, Abe T, et al. Critical role of PA28gamma in hepatitis C virus-associated steatogenesis and hepatocarcinogenesis. Proc Natl Acad Sci USA 2007;104:1661-1666.

29. Tsutsumi T, Suzuki T, Shimoike T, Suzuki R, Moriya K, Shintani $\mathrm{Y}$, et al. Interaction of hepatitis $\mathrm{C}$ virus core protein with retinoid $\mathrm{X}$ receptor alpha modulates its transcriptional activity. Hepatology 2002;35:937-946.

30. Mori Y, Moriishi K, Matsuura Y. Hepatitis C virus core protein: its coordinate roles with PA28gamma in metabolic abnormality and carcinogenicity in the liver. Int J Biochem Cell Biol 2008;40:1437-1442.

31. Fukasawa M, Tanaka Y, Sato S, Ono Y, Nitahara-Kasahara Y, Suzuki T, et al. Enhancement of de novo fatty acid biosynthesis in hepatic cell line Huh7 expressing hepatitis C virus core protein. Biol Pharm Bull 2006;29:1958-1961.

32. van der Poorten D, Shahidi M, Tay E, Sesha J, Tran K, McLeod $\mathrm{D}$, et al. Hepatitis $\mathrm{C}$ virus induces the cannabinoid receptor 1 . PLoS One 2010;5. pii: e12841.

33. Miyoshi H, Moriya K, Tsutsumi T, Shinzawa S, Fujie H, Shintani $\mathrm{Y}$, et al. Pathogenesis of lipid metabolism disorder in hepatitis C: polyunsaturated fatty acids counteract lipid alterations induced by the core protein. J Hepatol 2011;54:432-438.

34. Mancone C, Montaldo C, Santangelo L, Di Giacomo C, Costa $\mathrm{V}$, Amicone L, et al. Ferritin heavy chain is the host factor responsible for $\mathrm{HCV}$-induced inhibition of apoB-100 production and is required for efficient viral infection. J Proteome Res 2012;11:2786-2797.

35. Tachi Y, Katano Y, Honda T, Hayashi K, Ishigami M, Itoh $\mathrm{A}$, et al. Impact of amino acid substitutions in the hepatitis $\mathrm{C}$ virus genotype $1 \mathrm{~b}$ core region on liver steatosis and hepatic oxidative stress in patients with chronic hepatitis C. Liver Int 2010;30:554-559.

36. Akuta N, Suzuki F, Hirakawa M, Kawamura Y, Yatsuji H, Sezaki $\mathrm{H}$, et al. Amino acid substitutions in the hepatitis $\mathrm{C}$ virus core region of genotype $1 \mathrm{~b}$ are the important predictor of severe insulin resistance in patients without cirrhosis and diabetes mellitus. J Med Virol 2009;81:1032-1039.

37. Sumida Y, Kanemasa K, Hara T, Inada Y, Sakai K, Imai S, et al. Impact of amino acid substitutions in hepatitis $\mathrm{C}$ virus genotype $1 \mathrm{~b}$ core region on liver steatosis and glucose tolerance in noncirrhotic patients without overt diabetes. J Gastroenterol Hepatol 2011;26:836-842. 
38. Depla M, Uzbekov R, Hourioux C, Blanchard E, Le Gouge A, Gillet L, et al. Ultrastructural and quantitative analysis of the lipid droplet clustering induced by hepatitis $\mathrm{C}$ virus core protein. Cell Mol Life Sci 2010;67:3151-3161.

39. Jhaveri R, McHutchison J, Patel K, Qiang G, Diehl AM. Specific polymorphisms in hepatitis $\mathrm{C}$ virus genotype 3 core protein associated with intracellular lipid accumulation. J Infect Dis 2008;197:283-291.

40. Hourioux C, Patient R, Morin A, Blanchard E, Moreau A, Trassard S, et al. The genotype 3-specific hepatitis $\mathrm{C}$ virus core protein residue phenylalanine 164 increases steatosis in an in vitro cellular model. Gut 2007;56:1302-1308.

41. Cai T, Dufour JF, Muellhaupt B, Gerlach T, Heim M, Moradpour D, et al. Viral genotype-specific role of PNPLA3, PPARG, MTTP, and IL28B in hepatitis C virus-associated steatosis. J Hepatol 2011;55:529-535.

42. Ohnishi M, Tsuge M, Kohno T, Zhang Y, Abe H, Hyogo H, et al. IL28B polymorphism is associated with fatty change in the liver of chronic hepatitis C patients. J Gastroenterol 2012;Feb 18. [Epub ahead of print]PMID: 22350701.

43. Thompson AJ, Muir AJ, Sulkowski MS, Ge D, Fellay J, Shianna $\mathrm{KV}$, et al. Interleukin-28B polymorphism improves viral kinetics and is the strongest pretreatment predictor of sustained virologic response in genotype 1 hepatitis C virus. Gastroenterology 2010;139:120-129.

44. Clément S, Peyrou M, Sanchez-Pareja A, Bourgoin L, Ramadori P, Suter D, et al. Down-regulation of phosphatase and tensin homolog by hepatitis $\mathrm{C}$ virus core $3 \mathrm{a}$ in hepatocytes triggers the formation of large lipid droplets. Hepatology 2011;54:38-49.

45. Wedemeyer I, Bechmann LP, Odenthal M, Jochum C, Marquitan G, Drebber U, et al. Adiponectin inhibits steatotic CD95/Fas upregulation by hepatocytes: therapeutic implications for hepatitis C. J Hepatol 2009;50:140-149.
46. Piodi A, Chouteau P, Lerat H, Hézode C, Pawlotsky JM. Morphological changes in intracellular lipid droplets induced by different hepatitis $\mathrm{C}$ virus genotype core sequences and relationship with steatosis. Hepatology 2008;48:16-27.

47. Bernsmeier C, Duong FH, Christen V, Pugnale P, Negro F, Terracciano L, et al. Virus-induced over-expression of protein phosphatase $2 \mathrm{~A}$ inhibits insulin signalling in chronic hepatitis $\mathrm{C}$. J Hepatol 2008;49:429-440.

48. Kim KH, Shin HJ, Kim K, Choi HM, Rhee SH, Moon HB, et al. Hepatitis $B$ virus $X$ protein induces hepatic steatosis via transcriptional activation of SREBP1 and PPARgamma. Gastroenterology 2007;132:1955-1967.

49. Mankouri J, Tedbury PR, Gretton S, Hughes ME, Griffin SD, Dallas ML, et al. Enhanced hepatitis $\mathrm{C}$ virus genome replication and lipid accumulation mediated by inhibition of AMP-activated protein kinase. Proc Natl Acad Sci USA 2010;107:11549-11554.

50. Yang W, Hood BL, Chadwick SL, Liu S, Watkins SC, Luo G, et al. Fatty acid synthase is up-regulated during hepatitis $\mathrm{C}$ virus infection and regulates hepatitis $\mathrm{C}$ virus entry and production. Hepatology 2008;48:1396-1403.

51. Durante-Mangoni E, Zampino R, Marrone A, Tripodi MF, Rinaldi L, Restivo L, et al. Hepatic steatosis and insulin resistance are associated with serum imbalance of adiponectin/tumour necrosis factor-alpha in chronic hepatitis $\mathrm{C}$ patients. Aliment Pharmacol Ther 2006;24:1349-1357.

52. Shi ST, Polyak SJ, Tu H, Taylor DR, Gretch DR, Lai MM. Hepatitis C virus NS5A colocalizes with the core protein on lipid droplets and interacts with apolipoproteins. Virology 2002;292:198-210. 